\title{
Effective gravitational equations on brane world with induced gravity described by $f(R)$ term
}

\author{
Joel Saavedra and Yerko Vásquez \\ Instituto de Física, Pontificia Universidad Católica de Valparaíso, Casilla 4950, Valparaíso, Chile.
}

(Dated: August 9, 2021)

\begin{abstract}
In this article we study a generalization of Dvali-Gabadadze-Porrati scenarios, where the effective theory of gravity induced on the brane is given by a $f(R)$ term. We obtain the effective gravitational equations and the effective FLRW cosmological equation on the brane of this model. We show that this generalization has also two regime, a $5 D$ regime a low energies that has a self-accelerated branch of interest for cosmology and a $4 D$ regime at high energies that it is described a modified gravitational theory.
\end{abstract}

PACS numbers: $98.80 . \mathrm{Cq}$

\section{INTRODUCTION}

The idea of extra-dimensional theories have a long history that begins with an original idea of Kaluza and Klein [1] and finds a new realization within the modern string theory [2, 3]. In particular, the Randall-Sundrum scenario has acquired a great attention in the last decade [4, 5]. From the cosmological point of view, brane worlds offer a novel approach to our understanding of the evolution of the universe, for instance see Ref. [6] and the references therein. On the other hand, Dvali-Gabadadze-Porrati (DGP) brane world model [7], whose gravity behaves as four-dimensional at short distance scale but it shows higher-dimensional nature at larger distances, has attracted a great interest in the last time $[8,[9,10]$. This brane world model is characterized by the brane on which the fields of the standard model are confined, and contains the induced Einstein-Hilbert term. It also exhibits several cosmological features [11, 12, 13]. For example, in Ref. 13], the authors consider a five-dimensional bulk and obtain an accelerated expansion of the universe at late epoch, without need to introduce the cosmological constant. Therefore, the DGP model can be seen as providing a new mechanism to explain the late acceleration of the universe, based on a modification of gravitational theory [14] that arises from the extrinsic curvature. This new mechanics to explain cosmological late acceleration it is more interesting than introduce an exotic context of matter (Dark energy problem) and it is able to explain the origin of dark energy from the gravitational theory. However if we accept DGP as our gravitational theory we still have some problem in order to explain all history of the universe and match in a smooth way different phases in the evolution of the Universe. In this sense modified gravity emerge as serious candidate that it is able to addressing the definitive answers to several fundamental question about of dark energy [15]. For example, the origin of dark energy may be explained by term could be relevant at late times. Also can be considered as the source of early time inflation. Therefore modified gravity it is a natural scenario to have a theory that unified and explain both, the inflationary paradigm and the dark energy problem. Besides, modified gravity is expected to be useful in high energy physics. For a complete list of the main virtues of modified gravity see Ref. [16] were authors reviewed various modified gravities as gravitational alternative for dark energy. Specifically, they considered the versions of $f(R), f(G)$ or $f(R, G)$ gravity, model with non-linear gravitational coupling or string-inspired model with Gauss-Bonnet-dilaton coupling in the late universe where they lead to cosmic speed-up, however all models were considered in four dimensions. Another remarkable point is that at the intermediate epoch the gravity may be approximated by General Relativity. In this sense it is interesting to consider both: the DGP model and modified gravity as an unified scenario that we hope could be able to describe the majority of cosmological puzzles: the late acceleration, unification of early acceleration (inflation) with dark energy, also unification of the dark matter and dark energy and maybe a natural scheme that provide a mechanism in order to describes the transition from decceleration to acceleration in the universe evolution. However, in the DGP model, the bulk is a flat Minkowski space-time, but a reduced gravity term appears on the brane without tension. The origin of this reduced term, proportional to the Ricci Scalar (case of induced gravity), is understood as coming from from quantum effects of matter fields. In this sense, these terms should represent only the leading pieces of an effective action, that could also contain higher-order terms in a derivative expansion and higher powers of curvature tensors on the brane, such as $R^{2}$. Such nonlinear terms are generically suppressed by extra powers of the AdS curvature length scale, $l$, thus at distances much larger than $l$, we expect that these higher-order terms in the brane action could be neglected. This kind of modification was considered in Refs. [17] where creation of the inflationary brane universe in 5d bulk Einstein and Einstein-Gauss-Bonnet gravity was considered which one surface term where terms containing higher powers of the curvature, and recently in Ref. [18], where a brane with a scalar field and curvature corrections in DGP brane cosmology has been studied and projected equations on the brane were obtained using a Friedmann line element in the bulk. The plan of the paper is as follows: In Sec. II, we 
derive the effective equations on the brane, as a summary of the method described in Refs. [19] and 20]. In Sec. III, we derive the effective equations on the brane for modified induced gravity. In Sec. IV, we obtain the effective FLRW cosmological equation on the brane. Finally, we present conclusions in Sec. IV.

\section{EFFECTIVE EQUATIONS ON THE BRANE; THE METHOD}

In this section we summarize the main results of the Shiromizu-Maeda-Sasaki approach [19]. This derivation was generalized in Ref. 22], where was obtained the effective field equations on and off a 3-brane, to the case where an arbitrary energy-momentum tensor in the bulk is considered. In this brane world scenario, the observable universe is a $4 D$ surface, the brane, $M$, endowed with the metric $g_{\mu \nu}$ (Greek indices run over $0,1,2,3$ ), that is embedded in a five-dimensional space-time, the bulk, $V$, described by the metric $g_{A B}^{(5)}$ (capital indices run over $0,1,2,3,4$ ). The Standard Model particles and fields are confined to the brane, whereas gravity is free to propagate to the bulk. The induced metric on the brane is given by $g_{A B}=g_{A B}^{(5)}-n_{A} n_{B}$, where $n_{A}$ is the space-like unit vector normal to $M$. In this approach, the brane and bulk metrics remain general. The main idea is to project the five-dimensional Einstein equations to the brane and obtain the effective equations on the brane.

We consider the following action,

$$
S=S_{b u l k}+S_{\text {brane }}
$$

where $S_{b u l k}$ is the Einstein-Hilbert action in five-dimensional space-time and the brane action is

$$
S_{\text {brane }}=\int_{M} d^{4} x \sqrt{-g}\left(\frac{1}{k_{5}^{2}} K^{ \pm}+L_{\text {brane }}\right)
$$

Here, $k_{5}$ is the five-dimensional gravitational constant, $K^{ \pm}$is the trace of the extrinsic curvature where + and denote different sides of the brane and $L_{\text {brane }}$ is the effective four-dimensional Lagrangian.

Let $y$ be a Gaussian normal coordinate orthogonal to the brane, such that the hypersurface $y=0$ coincides with the brane and $n_{\mu} d x^{\mu}=d y$. Thus, the five-dimensional metric in terms of the induced metric on the brane is (locally) given by

$$
d s^{2}=g_{\mu \nu} d x^{\mu} d x^{\nu}+d y^{2} .
$$

The five-dimensional Einstein equation (including explicitly the contribution of the brane) determine the fivedimensional curvature tensor

$$
R_{A B}^{(5)}-\frac{1}{2} g_{A B}^{(5)} R^{(5)}=k_{5}^{2}\left[T_{A B}^{(5)}+\tau_{A B} \delta(y)\right]
$$

where $T_{A B}^{(5)}$ is the energy-momentum tensor of bulk matter fields,

$$
T_{A B}^{(5)}=-2 \frac{\delta^{(5)} L_{m}}{\delta^{(5)} g^{A B}}+g_{A B}^{(5)} L_{m}^{(5)},
$$

and $\tau_{A B}=g_{A}^{\mu} g_{B}^{\nu} \tau_{\mu \nu}$ and $g_{A}^{\mu}=\delta_{A}^{\mu}$, is the effective energy-momentum tensor localized on the brane,

$$
\tau_{\mu \nu}=-2 \frac{\delta L_{\text {brane }}}{\delta g^{\mu \nu}}+g_{\mu \nu} L_{\text {brane }} .
$$

The delta function in (4) ensures that the Standard Model fields are confined to the brane.

The Gauss equation gives the four-dimensional curvature tensor in terms of the projection of the five-dimensional curvature, with extrinsic curvature corrections,

$$
{ }^{(4)} R_{\beta \gamma \delta}^{\alpha}={ }^{(5)} R_{B C D}^{A} g_{A}^{\alpha} g_{\beta}^{B} g_{\gamma}^{C} g_{\delta}^{D}+K_{\gamma}^{\alpha} K_{\beta \delta}-K_{\delta}^{\alpha} K_{\beta \gamma},
$$

where the extrinsic curvature of $M$ is denoted by $K_{\mu \nu}=g_{\mu}^{A} g_{\nu}^{B} \nabla_{A} n_{B}$ and its trace is $K=K_{\mu}^{\mu}$. The Codazzi equation determines the change of $K_{\mu \nu}$ along the brane as

$$
D_{\nu} K_{\mu}^{\nu}-D_{\mu} K={ }^{(5)} R_{A B} n^{B} g_{\mu}^{A}
$$


where $D_{\nu}$ is the covariant derivative with respect to $g_{\mu \nu}$. The five-dimensional curvature tensor can be decomposed into the (traceless) Weyl tensor $C_{A B C D}^{(5)}$ and the Ricci part, as

$$
R_{A B C D}^{(5)}=\frac{2}{3}\left(g_{A[C}^{(5)} R_{D] B}^{(5)}-g_{B[C}^{(5)} R_{D] A}^{(5)}\right)-\frac{1}{6} g_{A[C}^{(5)} g_{D] B}^{(5)} R^{(5)}+C_{A B C D}^{(5)}
$$

Therefore, it follows that the induced Einstein tensor in four dimensions ${ }^{(4)} G_{\mu \nu}$ has the form

$$
\begin{aligned}
{ }^{(4)} G_{\mu \nu}=\frac{2 k_{5}^{2}}{3} & {\left[T_{R S}^{(5)} g_{\mu}^{R} g_{\nu}^{S}+\left(T_{R S}^{(5)} n^{R} n^{S}-\frac{1}{4} T^{(5)}\right) g_{\mu \nu}\right]+K K_{\mu \nu}-K_{\mu}^{\sigma} K_{\nu \sigma} } \\
& -\frac{1}{2} q_{\mu \nu}\left(K^{2}-K^{\alpha \beta} K_{\alpha \beta}\right)-E_{\mu \nu}
\end{aligned}
$$

where the projection of the bulk Weyl tensor to the surface orthogonal to $n^{A}$ is given by

$$
E_{\mu \nu}={ }^{(5)} C_{R N S}^{M} n_{M} n^{N} g_{\mu}^{R} g_{\nu}^{S}
$$

Integrating out the five-dimensional Einstein equation along the extra dimension from $y=-\epsilon$ to $y=+\epsilon$, and taking the limit $\epsilon \rightarrow 0$, leads to the Israel-Darmois junction conditions on the brane $\left[q_{\mu \nu}\right]=0$ and $\left[K_{\mu \nu}\right]=-k_{5}^{2}\left(\tau_{\mu \nu}-\frac{1}{3} g_{\mu \nu} \tau\right)$, where $[X]$ denotes the jump of $X$ over the brane, $[X]=\lim _{y \rightarrow+0} X-\lim _{y \rightarrow-0} X=X^{+}-X^{-}$. Furthermore, imposing the $\mathrm{Z}_{2}$-symmetry on the spacetime implies $K_{\mu \nu}^{-}=-K_{\mu \nu}^{+}$, so that we can use the junction condition equations to determine the extrinsic curvature on the brane as

$$
K_{\mu \nu}=-\frac{1}{2} k_{5}^{2}\left(\tau_{\mu \nu}-\frac{1}{3} g_{\mu \nu} \tau\right)
$$

Finally, substituting this equation into Eq. (10), we obtain the effective gravitational equations on the brane as

$$
G_{\mu \nu}=\frac{2 k_{5}^{2}}{3}\left[T_{R S}^{(5)} g_{\mu}^{R} g_{\nu}^{S}+g_{\mu \nu}\left(T_{R S}^{(5)} n^{R} n^{S}-\frac{1}{4} T^{(5)}\right)\right]+k_{5}^{4} \pi_{\mu \nu}-E_{\mu \nu},
$$

where the quadratic correction has the form

$$
\pi_{\mu \nu}=-\frac{1}{4} \tau_{\mu \alpha} \tau_{\nu}^{\alpha}+\frac{1}{12} \tau \tau_{\mu \nu}+\frac{1}{8} g_{\mu \nu} \tau_{\alpha \beta} \tau^{\alpha \beta}-\frac{1}{24} g_{\mu \nu} \tau^{2}
$$

and $E_{\mu \nu}$ is given by Eq. (11). The equations (11), (13) and (14) are equivalent to effective equations that were obtained in Ref. [19]. We assume that $L_{\text {brane }}$ includes additional gravitational contributions of the form of induced gravity on the brane. The only modification to take account in this case is, in the spirit of Ref. [20], correction to the effective energy momentum tensor $\tau_{\mu \nu}$. Recently, in Ref. [18], the effective equations on the brane were found, where the induced gravity was described by an arbitrary function $\mathcal{L}(R)$ of the Ricci scalar. In this article the effective equations were obtained by projection techniques. In the next section, we derive the effective equations on the brane where the induced gravity contains contributions of type $\mathcal{L}(R)$, using the methodology developed in Ref. [20].

\section{EFFECTIVE EQUATIONS ON THE BRANE; MODIFIED GRAVITY}

We consider induced gravity scenario that arises from higher-order corrections on the scalar curvature over the brane. This scenario corresponds to a version of the modified gravity on the DGP brane theory. Our Lagrangian on the brane is given by

$$
L_{\text {brane }}=\frac{\mu^{2}}{2} f(R)-\lambda+L_{m}
$$

where $\mu$ is a mass scale which may correspond to the 4-D Planck mass, $\lambda$ is a tension of the brane and $L_{m}$ presents the Lagrangian of the matter fields onto the brane. Higher-order correction to Einstein-Hilbert action typically arise in low energy effective actions of string theory models. It is well known that for $f(R)$ theories, if we make a conformal transformation $\bar{g}_{\mu \nu}=\Omega^{2}(x) g_{\mu \nu}$, are equivalent to Einstein-Hilbert action non- minimal coupled to a selfinteracting scalar field which a potential obtained from the conformal transformation. However, in our case situation is more complicated because $f(R)$ term lives only on the brane, and the conformal transformation should involves four dimensional sector in order to change just the induce gravity. At this point, in Ref. 21] was studied DGP inspired 
braneworld scenario where a scalar field nonminimally coupled to the induced Ricci curvature is present on the brane. Following the method discussed in the previous section, we define the energy momentum tensor as

$$
\tau_{\mu \nu}=-2 \frac{\delta L_{\text {brane }}}{\delta g^{\mu \nu}}+g_{\mu \nu} L_{\text {brane }} .
$$

Using Eq. (15), the energy momentum tensor reads

$$
\tau_{\mu \nu}=-\Lambda(R) g_{\mu \nu}+T_{\mu \nu}-\Sigma(R) G_{\mu \nu}+D_{\mu \nu},
$$

where the functions $\Lambda(R), \Sigma(R)$ and $D_{\mu \nu}$ are given by

$$
\Lambda(R)=\frac{\mu^{2}}{2}\left[R \frac{d f}{d R}-f(R)+2 \frac{\lambda}{\mu^{2}}\right]
$$

and

$$
\Sigma(R)=\mu^{2} \frac{d f}{d R}
$$

and

$$
D_{\mu \nu}=\mu^{2}\left[D_{\mu} D_{\nu}\left(\frac{d f}{d R}\right)-g_{\mu \nu} D^{\alpha} D_{\alpha}\left(\frac{d f}{d R}\right)\right]
$$

In this case, the quadratic corrections to the Einstein equations on the brane are written as follow,

$$
\begin{aligned}
\pi_{\mu \nu}= & -\frac{1}{12} \Lambda^{2}(R) g_{\mu \nu}+\frac{1}{6} \Lambda(R) T_{\mu \nu}-\frac{1}{6} \Lambda(R) \Sigma(R) G_{\mu \nu}+\frac{1}{6} D_{\mu \nu}+\Sigma(R)^{2} \pi_{\mu \nu}^{(G)}+\pi_{\mu \nu}^{(T)}+ \\
& +\pi_{\mu \nu}^{(D)}+\Sigma(R) G^{\rho \sigma} \mathcal{K}_{\mu \nu \rho \sigma}^{(T)}+\left(\Sigma(R) G^{\rho \sigma}-T^{\rho \sigma}\right) \mathcal{K}_{\mu \nu \rho \sigma}^{(D)},
\end{aligned}
$$

where we introduced the following definitions,

$$
\pi_{\mu \nu}^{(G)}=-\frac{1}{4} G_{\mu \alpha} G_{\nu}^{\alpha}+\frac{1}{12} G G_{\mu \nu}+\frac{1}{8} g_{\mu \nu} G_{\alpha \beta} G^{\alpha \beta}-\frac{1}{24} g_{\mu \nu} G^{2},
$$

and

$$
\begin{aligned}
& \pi_{\mu \nu}^{(T)}=-\frac{1}{4} T_{\mu \alpha} T_{\nu}^{\alpha}+\frac{1}{12} T T_{\mu \nu}+\frac{1}{8} g_{\mu \nu} T_{\alpha \beta} T^{\alpha \beta}-\frac{1}{24} g_{\mu \nu} T^{2}, \\
& \pi_{\mu \nu}^{(D)}=-\frac{1}{4} D_{\mu \alpha} D_{\nu}^{\alpha}+\frac{1}{12} D D_{\mu \nu}+\frac{1}{8} g_{\mu \nu} D_{\alpha \beta} D^{\alpha \beta}-\frac{1}{24} g_{\mu \nu} D^{2},
\end{aligned}
$$

and

$$
\begin{aligned}
\mathcal{K}_{\mu \nu \rho \sigma}^{(T)}= & \frac{1}{4}\left(g_{\rho \mu} T_{\sigma \nu}+g_{\sigma \nu} T_{\rho \mu}-g_{\mu \nu} T_{\rho \sigma}\right)+ \\
& +\frac{1}{12}\left(T\left(g_{\mu \nu} g_{\rho \sigma}-g_{\rho \mu} g_{\sigma, \nu}\right)-T_{\mu \nu} g_{\rho \sigma}\right),
\end{aligned}
$$

and

$$
\begin{aligned}
\mathcal{K}_{\mu \nu \rho \sigma}^{(D)}= & \frac{1}{4}\left(g_{\rho \mu} D_{\sigma \nu}+g_{\sigma \nu} D_{\rho \mu}-g_{\mu \nu} D_{\rho \sigma}\right)+ \\
& +\frac{1}{12}\left(D\left(g_{\mu \nu} g_{\rho \sigma}-g_{\rho \mu} g_{\sigma \nu}\right)-D_{\mu \nu} g_{\rho \sigma}\right),
\end{aligned}
$$

where $T$ is the trace of energy momentum tensor and $D$ is

$$
D=g^{\mu \nu} D_{\mu \nu}=-3 \mu^{2} D^{\mu} D_{\mu}\left(\frac{d f}{d R}\right) .
$$

Finally, replacing the quadratic corrections in the effective Einstein equations (13), we obtain 


$$
\begin{aligned}
\left(1+\frac{1}{6} k_{5}^{4} \Lambda(R) \Sigma(R)\right) G_{\mu \nu}= & -\frac{k_{5}^{2}}{2} \Lambda^{(5)} g_{\mu \nu}-\frac{1}{12} k_{5}^{2} \Lambda(R)^{2} g_{\mu \nu}+\frac{1}{6} k_{5}^{4} \Lambda(R) T_{\mu \nu}+\frac{1}{6} k_{5}^{4} D_{\mu \nu}+k_{5}^{4} \Sigma(R)^{2} \pi_{\mu \nu}^{(G)}+k_{5}^{4} \pi_{\mu \nu}^{(T)}+ \\
& +k_{5}^{4} \pi_{\mu \nu}^{(D)}+k_{5}^{4} \Sigma(R) G^{\rho \sigma} \mathcal{K}_{\mu \nu \rho \sigma}^{(T)}+k_{5}^{4}\left(\Sigma(R) G^{\rho \sigma}-T^{\rho \sigma}\right) \mathcal{K}_{\mu \nu \rho \sigma}^{(D)}-E_{\mu \nu},
\end{aligned}
$$

and the effective cosmological constant on the brane is given by

$$
\Lambda^{\prime}=\frac{k_{5}^{2}}{2}\left(\Lambda^{(5)}+\frac{1}{6} \Lambda(R)^{2} k_{5}^{2}\right)
$$

note that these equations are exactly the same effective equations as in the reference [20], when $f(R)=R$.

\section{EFFECTIVE FRW COSMOLOGICAL EQUATION ON THE BRANE}

Now we are interested in computing the effective cosmological equations on the brane. We discuss the FriedmannRobertson-Walker universes filled with a perfect fluid. First we consider the five-dimensional metric described by the interval

$$
d s^{2}=d \chi^{2}+g_{\mu \nu} d x^{\mu} d x^{\nu}
$$

and the energy momentum tensor is given by $T_{A B}^{(5)}=\frac{-\Lambda^{(5)}}{k_{5}^{2}} g_{A B}^{(5)}$. We decompose the metric as

$$
g_{A B}^{(5)}=\left(\begin{array}{cc}
g_{\mu \nu} & 0 \\
0 & 1
\end{array}\right)
$$

so that its inverse has the form

$$
g^{(5) A B}=\left(\begin{array}{cc}
g^{\mu \nu} & 0 \\
0 & 1
\end{array}\right)
$$

and the normal vector to the brane is $n^{R}=(0,0,0,0,1)$. Therefore, it is not hard to show that the effective equations (13) reduce to

$$
\frac{2}{3} k_{5}^{2}\left[T_{R S}^{(5)} g_{\mu}^{R} g_{\nu}^{S}+g_{\mu \nu}\left(T_{R S}^{(5)} n^{R} n^{S}-\frac{1}{4} T^{(5)}\right)\right]=-\frac{1}{2} \Lambda^{(5)} g_{\mu \nu}
$$

and therefore the basic equations read as follows,

$$
\begin{aligned}
& G_{0}^{0}=-\frac{1}{2} \Lambda^{(5)}+k_{5}^{4} \pi_{0}^{0}-E_{0}^{0}, \\
& G_{j}^{i}=-\frac{1}{2} \Lambda^{(5)} \delta_{j}^{i}+k_{5}^{4} \pi_{j}^{i}-E_{j}^{i} .
\end{aligned}
$$

On the other hand, from the geometry of Friedmann-Robertson-Walker universes, it is well-known that the components of the Einstein tensor are given by the following expressions,

$$
\begin{aligned}
& G_{0}^{0}=-3\left(H^{2}+\frac{k}{a^{2}}\right), \\
& G_{j}^{i}=-\left(2 H^{\prime}+3 H^{2}+\frac{k}{a^{2}}\right) \delta_{j}^{i},
\end{aligned}
$$

where $H=\frac{\dot{a}}{a}$ represents the Hubble constant, $a(t)$ is the scale factor and $k$ describes the hypersurfaces of homogeneity that could be represented as a three-sphere, a three-plane or a three-hyperboloid, with values $k=1,0,-1$, respectively. Since the space-time is isotropic and homogeneous, it is possible to show that $D^{\nu} \pi_{\mu \nu}=0$ and $D^{\nu} E_{\mu \nu}=0$ are satisfied [19], and the relevant components of $\pi_{\mu \nu}$ are given by

$$
\begin{aligned}
\pi_{0}^{0} & =-\frac{1}{12}\left(\tau_{0}^{0}\right)^{2} \\
\pi_{j}^{i} & =\frac{1}{12} \tau_{0}^{0}\left(\tau_{0}^{0}-2 \tau_{1}^{1}\right) \delta_{j}^{i} .
\end{aligned}
$$


The energy momentum tensor then becomes

$$
\tau_{\nu}^{\mu}=-\mu^{2} \frac{d f}{d R} G_{\nu}^{\mu}-\frac{\mu^{2}}{2}\left[R \frac{d f}{d R}-f(R)+2 \frac{\lambda}{\mu^{2}}\right] \delta_{\nu}^{\mu}+T_{\nu}^{\mu}+\mu^{2}\left[D^{\mu} D_{\nu}\left(\frac{d f}{d R}\right)-\delta_{\nu}^{\mu} D^{\alpha} D_{\alpha}\left(\frac{d f}{d R}\right)\right],
$$

where $T_{\nu}^{\mu}=\operatorname{diag}(-\rho, P, P, P)$. Combining 00 component of Eqs. (34), (35) and (37), solving the equation obtained for $H^{2}+k / a^{2}$, we obtain the effective basic cosmological equations on the brane,

$$
\begin{aligned}
H^{2}+\frac{k}{a^{2}}= & \frac{2}{\mu^{4} k_{5}^{4}\left(\frac{d f}{d R}\right)^{2}}+\frac{1}{3 \mu^{2}\left(\frac{d f}{d R}\right)}\left[\frac{\mu^{2}}{2}\left(R \frac{d f}{d R}-f(R)-6 H \dot{R} \frac{d^{2} f(R)}{d R^{2}}\right)+\lambda+\rho\right] \\
& \pm \frac{2}{k_{5}^{2} \mu^{2}\left(\frac{d f}{d R}\right)} \sqrt{\frac{1}{k_{5}^{4} \mu^{4}\left(\frac{d f}{d R}\right)^{2}}+\frac{\frac{\mu^{2}}{2}\left(R \frac{d f}{d R}-f(R)-6 H \dot{R} \frac{d^{2} f(R)}{d R^{2}}\right)+\lambda+\rho}{3 \mu^{2}\left(\frac{d f}{d R}\right)}-\frac{\Lambda^{(5)}}{6}-\frac{\varepsilon_{0}}{3 a^{4}}},
\end{aligned}
$$

where $\varepsilon_{0}$ is an integration constant. The above equation represents the effective Friedmann equation for the DGP theory with curvature corrections on the brane. Note that correct limit is obtained when $f(R)=R$. In order to study the different limits of Eq. (38), we combined 00 component of Eqs. (34), (35) and (37), then taking root-sqare of the obtained equation, we arrive to more manipulable form $[23]$

$$
r\left[\left(H^{2}+\frac{k}{a^{2}}\right) \frac{d f}{d R}\right]-\frac{k_{5}^{2}}{6}\left(\rho+\lambda+\frac{\mu^{2}}{2}\left(R \frac{d f}{d R}-f(R)-6 H \dot{R} \frac{d^{2} f(R)}{d R^{2}}\right)\right)= \pm \sqrt{H^{2}+\frac{k}{a^{2}}-\frac{1}{3} \frac{\varepsilon_{0}}{a^{4}}-\frac{1}{6} \Lambda^{(5)}},
$$

where $r$ is the cross-over scale and is defined as $r=\frac{1}{2} k_{5}^{2} \mu^{2}=\frac{1}{2} \frac{k_{5}^{2}}{k_{4}^{2}}$, and two different solutions ( \pm ) correspond to two different embedding of the brane within the bulk..

If we choose a Minkowskian bulk $\left(\Lambda^{(5)}=0, \varepsilon_{0}=0\right)$ with $\lambda=0$ on a flat brane $(k=0)$ we get

$$
r H^{2} \frac{d f}{d R}-\frac{k_{5}^{2}}{6}\left(\rho+\frac{\mu^{2}}{2}\left(R \frac{d f}{d R}-f(R)-6 H \dot{R} \frac{d^{2} f}{d R^{2}}\right)\right)= \pm H
$$

where for $f(R)=R$ (DGP standard model) we obtain the right limits, at low energies $\rho \longrightarrow 0$ appear the brach $\mathrm{DGP}(+)$ characterized by $H \longrightarrow \frac{1}{r}$ and the branch $\mathrm{DGP}(-)$ here $H \longrightarrow 0$. DGP $(+)$ branch is important from cosmological point of view, because this branch has a self-accelerated phase. Note that this self-acceleration in this limit can be seen in term of scale factor as an exponential expansion $a(t)=a_{0} e^{\frac{t}{r}}$. In order to explore cosmological consequence we are considering $f(R)=R^{m}$, and also we focus at low energies limit $(\rho \longrightarrow 0)$ in a Minkowskian bulk $\Lambda^{(5)}=0, \varepsilon_{0}=0$, and the case of flat brane with $\lambda=0$. We are asking if our theory allows a solution of the form $a(t)=a_{0} e^{\alpha\left(t-t_{0}\right)}$, where $\alpha$ is a constant parameter. Then if we replace our choice of $f(R)$ and the Ansatz of the scale factor in Eq.(40) ${ }^{1}$ we obtain,

$$
r 12^{m-1} \alpha^{2 m}(2-m)= \pm \alpha
$$

this equation allows to investigate behaviors of the solution in this modified DGP brane. First we consider the case $m=1$ (DGP standard brane), we obtain $H=\alpha=\frac{1}{r}$ positive branch and $H=\alpha=0$ for the negative branch(-), therefore we recover standard behaviors of DGP brane. In the case $m=2$ we obtain $H=\alpha=0$ in both branches static universe (like Einstein's universe). For $m>2$ we obtain two solutions for the Hubble parameter $H=\alpha=0$ the static branch and

$$
H=\alpha=\left(\frac{1}{(m-2) r 12^{m-1}}\right)^{\frac{1}{(2 m-1)}}
$$

for the accelerated branch. Therefore for $f(R)=R^{m}$ it is possible to obtain a model with self-acceleration for $m>0$ $(m \neq 2)$. The self-acceleration for the case with $m=2$, it is included if we choose $f(R)=R+\beta R^{m}$. In sum we obtain a self-accelerated cosmology at the low energy limit.

On the other hand for the high energies limit $H>>\frac{1}{r \frac{d f}{d R}}$ of Eq. (40)for a Minkowskian bulk $\Lambda^{(5)}=0, \varepsilon_{0}=0$, and the case of flat brane with $\lambda=0$, we obtain that both branches have the same limit

$$
H>>\frac{1}{r \frac{d f}{d R}}, \Longrightarrow H^{2}=\frac{1}{3 \mu^{2} \frac{d f}{d R}}\left(\rho+\lambda+\frac{\mu^{2}}{2}\left(R \frac{d f}{d R}-f(R)-6 H \dot{R} \frac{d^{2} f}{d R^{2}}\right)\right),
$$

\footnotetext{
${ }^{1}$ where we used that $R=12 H^{2}+6 \dot{H}=12 \alpha^{2}$
} 
where we recover the standard Friedmann equation if we take $f(R)=R$. We noticed that Eq. (43) is equal to one that is obtained from $4 D$ modified gravity [24]. Therefore the $4 \mathrm{D}$ regime is driven by a modified gravitational theory.

Finally as $R$ contains second derivatives of the scale factor $a(t)$ in Eq.(38), means that there are more degrees of freedom in the space of solutions than for Einstein gravity with a cosmological constant or the standard DGP model and it is needed to confront this theory with observational data. At this point, one interesting approach is to make a conformal transformation in to the four dimensional part of the theory. Where we can see explicitly the new degree of freedom thought the scalar field that appear in the transformed theory. This theory behaviors like a DGP model with a scalar field $(\phi)$ (where are disguise all the effect of the modified gravity in DGP theory) with a potential $V(\phi)$ evolving in FRW DGP brane where the matter is endowed with a non-minimal coupling set by $\phi$. Certainly, Eq. (38) encodes all cosmological information that we hope to discuss in the near future. In sum using the method developed in Ref. [20], we obtain the effective gravitational equations on the DGP brane world where the induced gravity was described by a general $f(R)$ term. Applying the formulas to cosmology -a universe filled with a perfect fluid-, we derive the generalized Friedmann equation on the brane. This modified Friedmann equation give us a new possibility for studying cosmological models through the inclusion of curvature corrections in the induced gravity term on the brane, and really open a new kind of model still unexplored. In this sense it is interesting to consider both: the DGP model and modified gravity as an unified scenario that we hope could be able to describe the majority of cosmological puzzles: the late acceleration, unification of early acceleration (inflation) with dark energy, also unification of the dark matter and dark energy and maybe a natural scheme that provide a mechanism in order to describes the transition from decceleration to acceleration in the universe evolution. . We note that our gravitational effective equations (13), as well as the Friedmann effective equation, have the correct limit when $f(R)=R$, and can be considered as a genuine generalization of DGP effective equations on modified DGP brane world. Whose high and low energies limits show a self-accelerated phase (interesting from the cosmological point of view) in the $5 D$ regime and a modified gravitational theory for the $4 D$ regime, respectively. Investigations of further cosmological implications, including the late acceleration of the universe, are under our current considerations.

\section{Acknowledgments}

We are grateful to A. N. Aliev,O. Mišković and S. D. Odintsov for enlightening comments. J.S. is supported by the COMISION NACIONAL DE CIENCIAS Y TECNOLOGIA through FONDECYT Grant N ${ }^{0}$. 11060515, and also was partially supported by PUCV Grant $\mathrm{N}^{0}$. $123.789 / 2007$. Y.V was partially supported by MINISTERIO DE EDUCACION through MECESUP Grants FSM 0204, by Dirección de Estudios Avanzados PUCV and by CONICYT Scholarship 2008.

[1] Kaluza, T. 1921 Sitzungsber.Preuss.Akad. Wiss.Berlin (Math.Phys.) K1 p 966; Klein O 1926 Z.Phys. 37895.

[2] A. Font and S. Theisen, Lect. Notes Phys. 668, 101 (2005).

[3] C. S. Chan, P. L. Paul and H. L. Verlinde, Nucl. Phys. B 581, 156 (2000).

[4] L. Randall and R. Sundrum, Phys. Rev. Lett. 83, 3370 (1999).

[5] L. Randall and R. Sundrum, Phys. Rev. Lett. 83, 4690 (1999).

[6] R. Maartens, Living Rev. Rel. 7, 7 (2004).

[7] G. R. Dvali, G. Gabadadze and M. Porrati, Phys. Lett. B 484, 112 (2000).

[8] S. del Campo and R. Herrera, Phys. Lett. B 653, 122 (2007)

[9] R. Gregory, N. Kaloper, R. C. Myers and A. Padilla, JHEP 0710, 069 (2007).

[10] A. Lue, Phys. Rept. 423, 1 (2006).

[11] G. R. Dvali, G. Gabadadze, M. Kolanovic and F. Nitti, Phys. Rev. D 64, 084004 (2001)

[12] C. Deffayet, G. R. Dvali, G. Gabadadze and A. I. Vainshtein, Phys. Rev. D 65, 044026 (2002).

[13] C. Deffayet, G. R. Dvali and G. Gabadadze, Phys. Rev. D 65, 044023 (2002).

[14] G. Gabadadze, Nucl. Phys. Proc. Suppl. 171, 88 (2007).

[15] S. Nojiri and S. D. Odintsov, J. Phys. Conf. Ser. 66, 012005 (2007) arXiv:hep-th/0611071.

[16] S. Nojiri and S. D. Odintsov, eConf C0602061, 06 (2006) [Int. J. Geom. Meth. Mod. Phys. 4, 115 (2007)]

[17] S. Nojiri and S. D. Odintsov, Gen. Rel. Grav. 37, 1419 (2005)

[18] K. Atazadeh and H. R. Sepangi, arXiv:0710.0214 [gr-qc].

[19] T. Shiromizu, K. i. Maeda and M. Sasaki, Phys. Rev. D 62, 024012 (2000).

[20] K. i. Maeda, S. Mizuno and T. Torii, Phys. Rev. D 68, 024033 (2003).

[21] K. Nozari, JCAP 0709, 003 (2007).

[22] A. N. Aliev and A. E. Gumrukcuoglu, Class. Quant. Grav. 21, 5081 (2004).

[23] R. A. Brown, arXiv:gr-qc/0701083 
[24] S. Fay, S. Nesseris and L. Perivolaropoulos, Phys. Rev. D 76, 063504 (2007) arXiv:gr-qc/0703006. 